\title{
Response measurements on an echinodome subjected to explosive loading
}

\author{
K.M.M. El-Deeb ${ }^{\mathrm{a}}$ and R. Royles ${ }^{\mathrm{b}, *}$ \\ ${ }^{a}$ Linear and Non-linear Structural Dynamics Limited, \\ Edinburgh, Scotland, UK \\ ${ }^{\mathrm{b}}$ School of Civil and Environmental Engineering, \\ Division of Engineering, The University of Edinburgh, \\ Crew Building, King's Buildings, West Mains Road, \\ Edingburgh EH9 3JN, Scotland, UK
}

Received 8 May 1996

Revised 4 January 1999

Free field explosion experiments were carried out to determine the general characteristics of the pressure pulse generated by a detonator underwater. An echinodome - an axisymmetric shell structure of optimum form - was subjected to a shock wave from the same type of detonator, the structure being in a floating submerged and tethered state. Some indication of the influence of the structure on the incident pressure wave was obtained from a surface mounted miniature pressure gage. Structural response was measured with the aid of strain gages from which principal stress data were deduced. Consideration was given to the theoretical modelling aspects of the problem.

Keywords: Underwater explosion, echinodome, electrical resistance strain gages, pressure gages

\section{Introduction}

When a spill of a refrigerant liquid becomes superheated to an extent that permits homogeneous nucleation to take place, an overpressure is produced and such a phenomenon is known as a rapid phase transition (RPT) [2].

Liquefied natural gas (LNG) is a hazardous liquid generally stored under high pressure, or at very low temperatures, or a combination of lower levels of both. It has been suggested previously [5], that such liquids could be stored underwater in echinodomes with marked safety benefits. If leakage took place from an

\footnotetext{
${ }^{*}$ Corresponding author.
}

underwater container the cold liquid would be superheated after contacting the surrounding medium (water) resulting in an RPT which resembles an explosion and consequently shock waves would be transmitted to nearby structures. Therefore it is necessary to consider RPT loading on underwater reservoirs such as echinodomes, containing highly volatile liquids at very low temperatures.

In this work underwater RPT events were simulated using small detonations whose characteristics were established from free field experimental studies, Fig. 1. Their effects on an echinodome prototype, Fig. 2, were examined using the arrangement shown in Fig. 3 in order to obtain strain history data which could be utilised in validation of fluid-structure interaction codes.

\section{Principal underwater explosion phenomena}

An explosion is a rapid release of energy in a considerably short time. Explosive materials undergo chemical reactions releasing gaseous materials at very high pressures and temperatures. In the following subsections the behaviour of the gaseous explosion products (often known as the bubble) is described. More details can be found elsewhere [1].

\subsection{Detonation process}

During the initial phase of the explosion phenomenon a detonation wave develops. The detonation wave propagating speed is a characteristic of each explosive material and is several times that of the subsequent shock wave.

\subsection{Shock wave}

At the boundary between the explosion gases and the surrounding water compression waves are generated, developing very steep fronts as they progress. Such waves are known as shock waves and they travel at an average velocity of $1400-1500 \mathrm{~m} / \mathrm{s}$ when they 


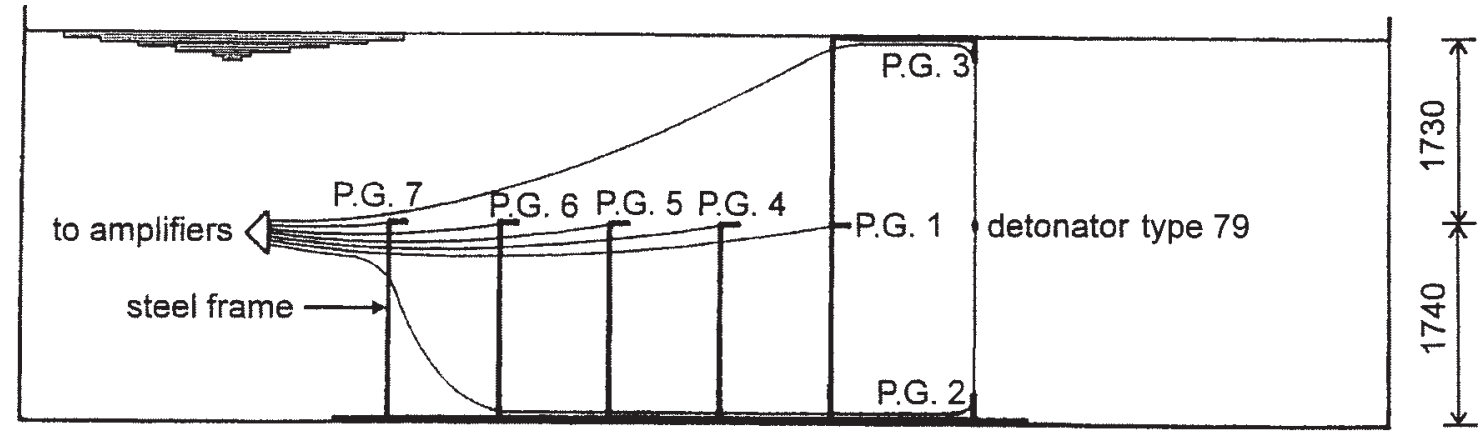

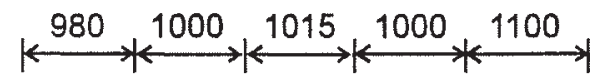

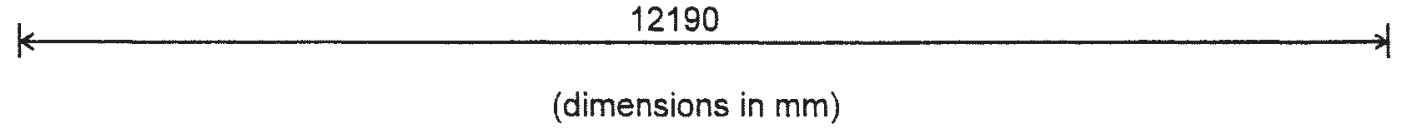

Fig. 1. Arrangement of pressure gages for underwater free field explosion tests.

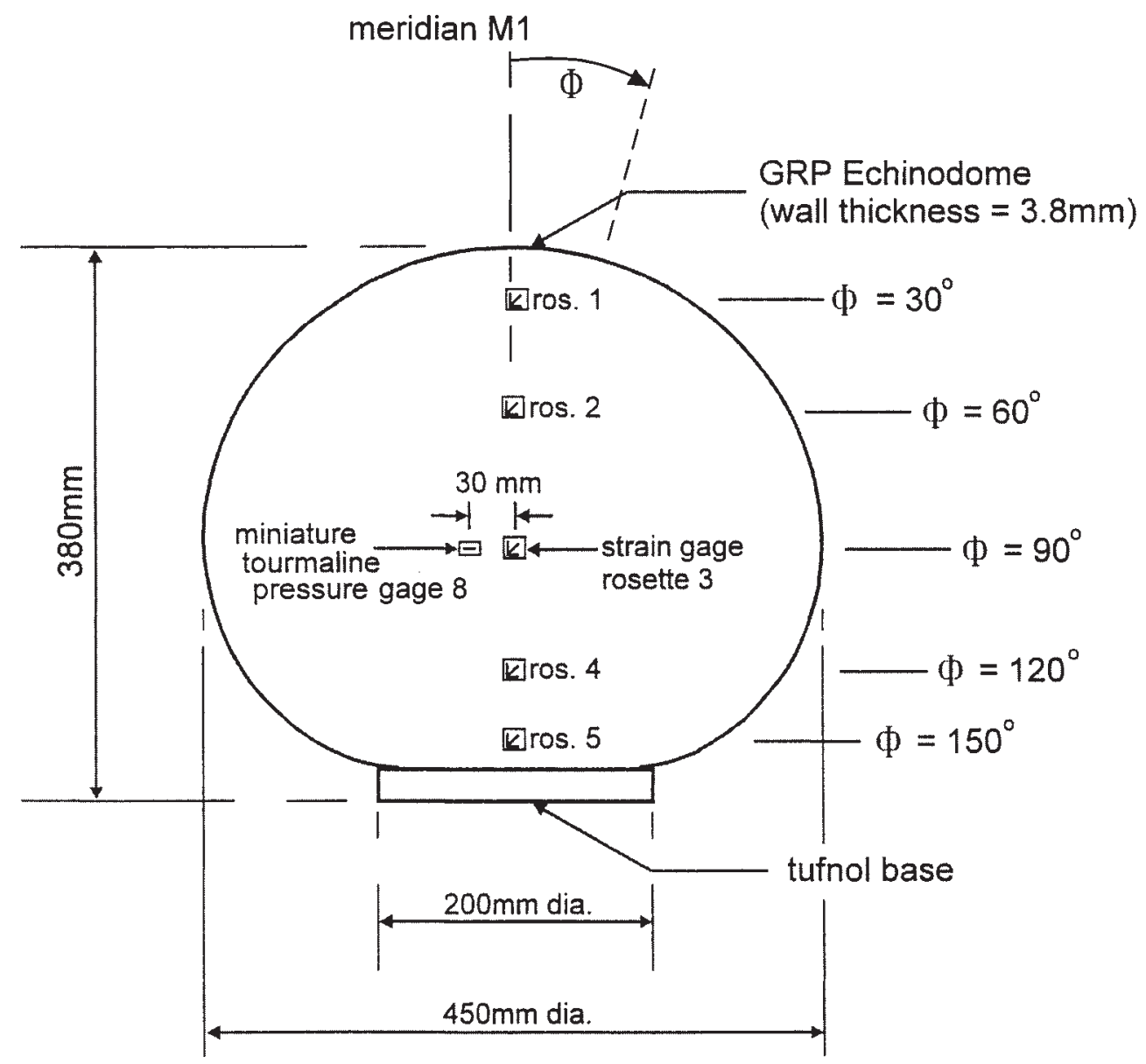

Fig. 2. Disposition of miniature tourmaline pressure gage and strain gage rosettes on shell surface. 


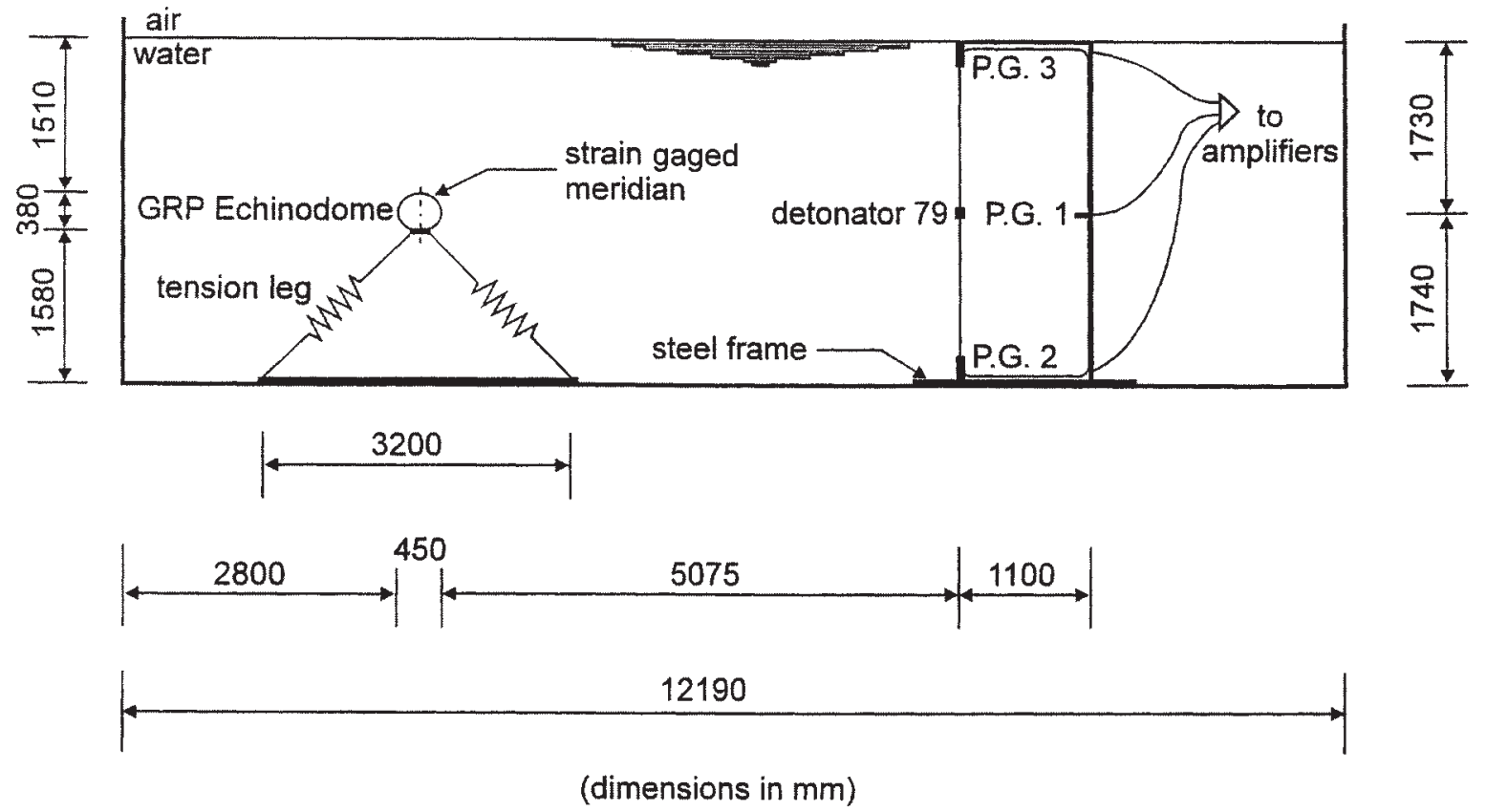

Fig. 3. Prototype location in water tank for underwater explosion tests ( $\mathrm{PG}=$ pressure gage).

are considerably distant from the charge. The time history of the pressure pulse is generally characterised by having a discontinuous rise followed by an exponential decay.

\subsection{Bubble pulse}

The gaseous products that are formed post the underwater explosion event, referred to as gas bubble, expand in the surrounding medium until the internal pressure drops below the ambient hydrostatic pressure. The bubble then starts to contract because of the higher surrounding pressure until it collapses at which time a secondary pulse, known as the bubble pulse, is emitted. Several cycles of expansion and contraction are repeated until the bubble energy is consumed, or the bubble vents at the free surface. The bubble characteristics (periodic time and maximum radius) are dependent on the hydrostatic pressure. In an infinite fluid medium the bubble migrates upwards due to buoyancy. In a semiinfinite fluid medium the bubble attracts to rigid surfaces and repels from free surfaces. Bubble loading is a combination of fluid flow, because of the displacement of large masses of water, and a bubble pulse which contains approximately the same amount of energy to that of the shock wave.

\section{Experimental approach}

The experimental investigation was divided into two parts. The first part comprised a set of free field experiments which were carried out in order to determine the pressure pulse characteristics (peak pressure, decay rate, impulse and energy) for a cylindrical charge of a specific mass at a given position. In the second part the cylindrical charge was detonated at a predetermined stand-off distance from the echinodome prototype described below.

The echinodome, whose meridional profile is shown in Fig. 2, was made from GRP using randomly orientated chopped strand mat with a $26 \%$ glass fraction in an epoxy matrix. The material was found to be linear elastic to fracture with the following characteristics:
Ultimate tensile strength
$55.4 \mathrm{MPa}$
Young's modulus of elasticity $\quad 8800 \mathrm{MPa}$
Poisson's ratio
0.36
Mass density
$1100 \mathrm{~kg} / \mathrm{m}^{3}$

\section{Charge design}

The extent of damage caused by an underwater explosion depends on the dimensions and characteristic (periodic) times of the structure upon which the gener- 

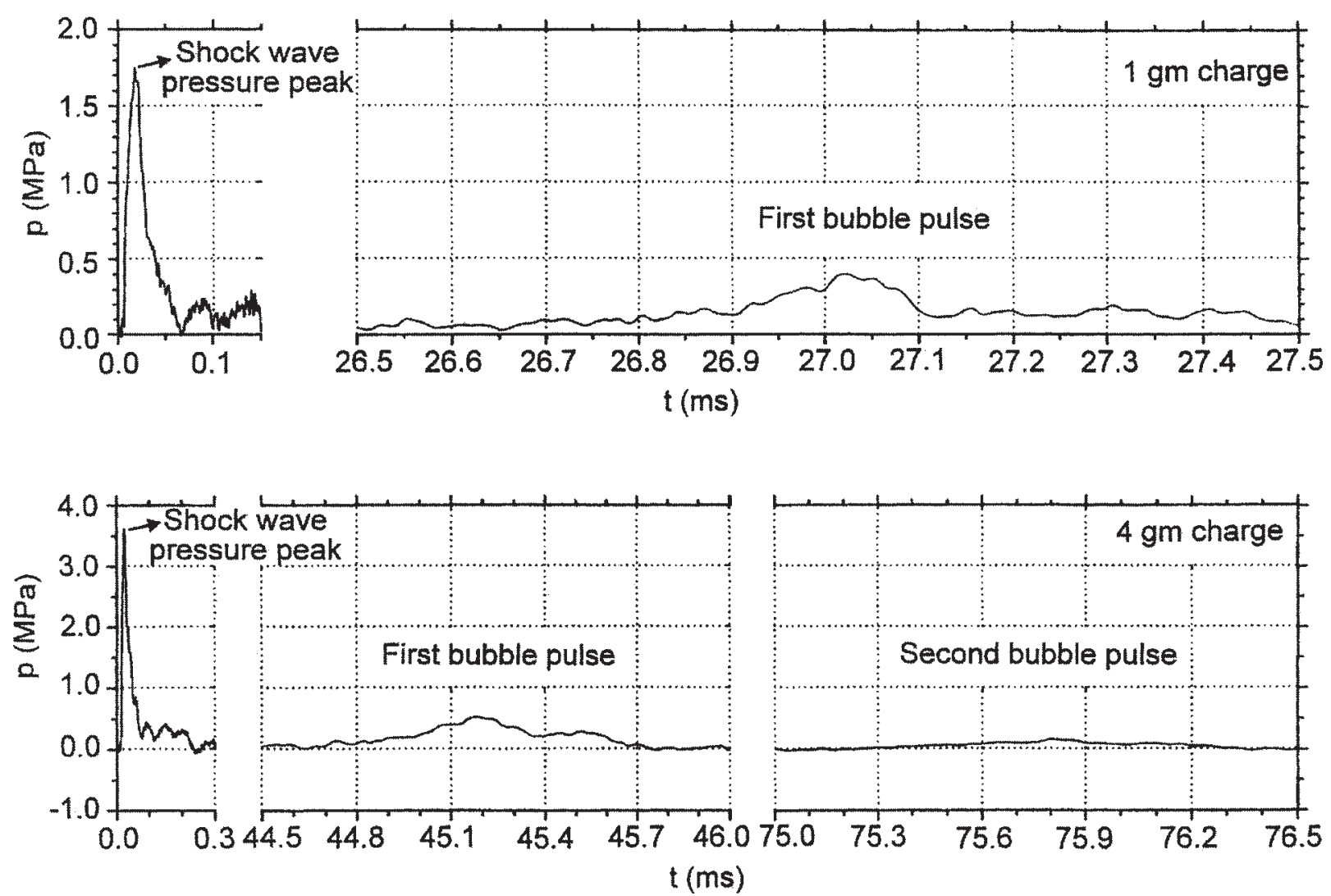

Fig. 4. Off side pressure-time history $1.535 \mathrm{~m}$ away from cylindrical charges of various mass (detonator type 79).

ated pressure wave acts. The time duration of the pulse relative to the structural periodic times is one of the parameters controlling the dynamic response to such transient loading. Cole [1] mentioned that if cavitation occurred in the water near the structure the resultant deformation would roughly be proportional to the square root of the shock wave energy flux density.

The variation of the peak pressure $\left(p_{\mathrm{m}}\right)$, impulse $(I)$ and energy $\left(E_{\mathrm{f}}\right)$ with the charge mass $(W)$ and standoff distance $(R)$ can be represented by empirical relationships which may be used to determine the upper bound charge size to ensure no structural damage.

This approach was adopted to establish the size of an explosive charge mass placed at $5 \mathrm{~m}$ from the target so that no geometric non-linearity of the echinodome prototype would result and at the same time would produce measurable strain levels.

The estimated charge mass was found to be equal to $1 \mathrm{~g}$ and a standard electric detonator type 79 was selected for the purpose of the current experimental study. The charge was of cylindrical shape.

Pulse shapes measured off the side of cylindrical charges are similar to those of spherical charges in that the discontinuous rise is followed by an exponential decay. However, the pressure-time curves differ in shape and peak level if measurements are made along the axis of symmetry, often possessing two peaks.

\section{Free field arrangement}

For a given charge size and type the pulse characteristics of the shock wave, in the form of peak pressure, decay rate or time duration, impulse and energy, vary with the stand-off distance. Consequently, a set of free field experiments were carried out in order to estimate such properties as well as to determine the pulse shape at the point where the shock wave would impinge the echinodome prototype in a later experimental set-up.

The changes in the pressure field surrounding detonator type 79 following detonation were measured utilising seven tube like tourmaline pressure gages. These gages can measure pressure levels of up to several hundred MPa. Calibration of the pressure gages was performed on site. The sensitivity of each gage was determined by applying a standard pressure pulse of 
6.88 $\mathrm{MPa}$ and measuring the corresponding voltage change using a digital oscilloscope.

A frame made of steel was erected on which the pressure gages were fixed as shown in Fig. 1. The frame was then lowered into a water tank of dimensions $12.190 \mathrm{~m} \times 6.100 \mathrm{~m}$ in a $3.470 \mathrm{~m}$ of water depth. The tank walls and base were made of reinforced concrete with thick steel plates lining it. The position of pressure gage 7 corresponded to that of the nearest point of the test structure to the explosive charge. Pressure gages 2 and 3 were used to determine the characteristics of the reflected shock wave from the bottom of the tank (rigid boundary) and the water surface (free boundary), respectively.

The free field test was repeated five times, using a $1 \mathrm{~g}$ charge on each occasion, to establish the scatter in the gathered data.

\section{Target set-up}

A different experimental set-up shown in Fig. 3, was used when testing the echinodome structure (in an empty state). A set of four cables was used to position the prototype at a depth of $1.525 \mathrm{~m}$ of water from its apex (the design pressure head of the test structure). Each cable was made of six strands and a single core. The four cables acted as tension legs supporting the test structure because of the buoyant forces acting upwards on the empty shell structure.

Part of the steel frame used in the free field experiment was again employed with some of the attached pressure gages to gain more confidence in the measured pressure pulses as well as to position the detonator at the same water depth as the maximum diameter of the echinodome prototype.

Structural response was measured using electrical resistance foil strain gage rosettes with each gage having a resistance of $350 \pm 1 \Omega, 3 \mathrm{~mm}$ gage length and an average gage factor equal to 2.15 . The rosettes were bonded on the outer surface of the shell at five different positions in the same meridional plane with angles $\phi=30^{\circ}, 60^{\circ}, 90^{\circ}, 120^{\circ}$ and $150^{\circ}$ away from the apex as depicted in Fig. 2. For each rosette one gage was aligned with the meridional profile, another along the corresponding parallel circle and the third in between them at an inclination of $45^{\circ}$. Voltage excitation of the strain gages, filtering and conditioning of the strain signals were carried out using special signal conditioning amplifier units.
The detonator was set at a position approximately $5 \mathrm{~m}$ from the prototype. The strain gage rosettes, the explosive charge and the three pressure gages were in the same vertical plane as shown in Fig. 3.

A newly developed miniature tourmaline pressure gage PCB model 105M114 was attached to the test structure at its maximum diameter $\left(\phi=90^{\circ}\right), 30 \mathrm{~mm}$ from the strain gage rosette lying on the same parallel circle and was nominated P.G.8. The gage had a maximum range of $6.88 \mathrm{MPa}$. Wax which was employed in the waterproofing process acted as a bonding agent between the transducer and the prototype.

Again five tests were carried out, using a $1 \mathrm{~g}$ charge in four tests and a $4 \mathrm{~g}$ charge in one test. The former were simple repetitions to give check on statistical quality.

\section{Signal processing}

Analogue pressure and strain signals were digitised and then processed using the software DADISP, version 1.05 (1987). DADISP enabled averaging of signals, d.c. shift removal, calibration, filtering, discrete Fourier transformation and many more mathematical applications.

A block diagram showing the instrumentation employed in the current experimental investigation for the data acquisition and analysis stages is presented in Fig. 5.

The signal processing stage comprised the following five main steps:

(i) Common triggering. Before performing any averaging process on any ensemble of time domain signals it was important to adjust the trigger points to have the same time of occurrence.

(ii) Ensemble averaging. Time domain signals were averaged in order to reduce inherent random errors in the form of noise.

(iii) Offset removal. D.c. offsets were determined by averaging all sample points prior to trigger. Then such transitions were subtracted from the corresponding ensemble averaged signals.

(iv) Digital filtering. Low pass filters were designed to be applied to pressure and strain signals with the purpose of attenuating the high frequency components.

(v) Calibration. All digital signals were scaled by the corresponding calibration constants to transform their amplitudes from voltage units to pressure or strain units. 


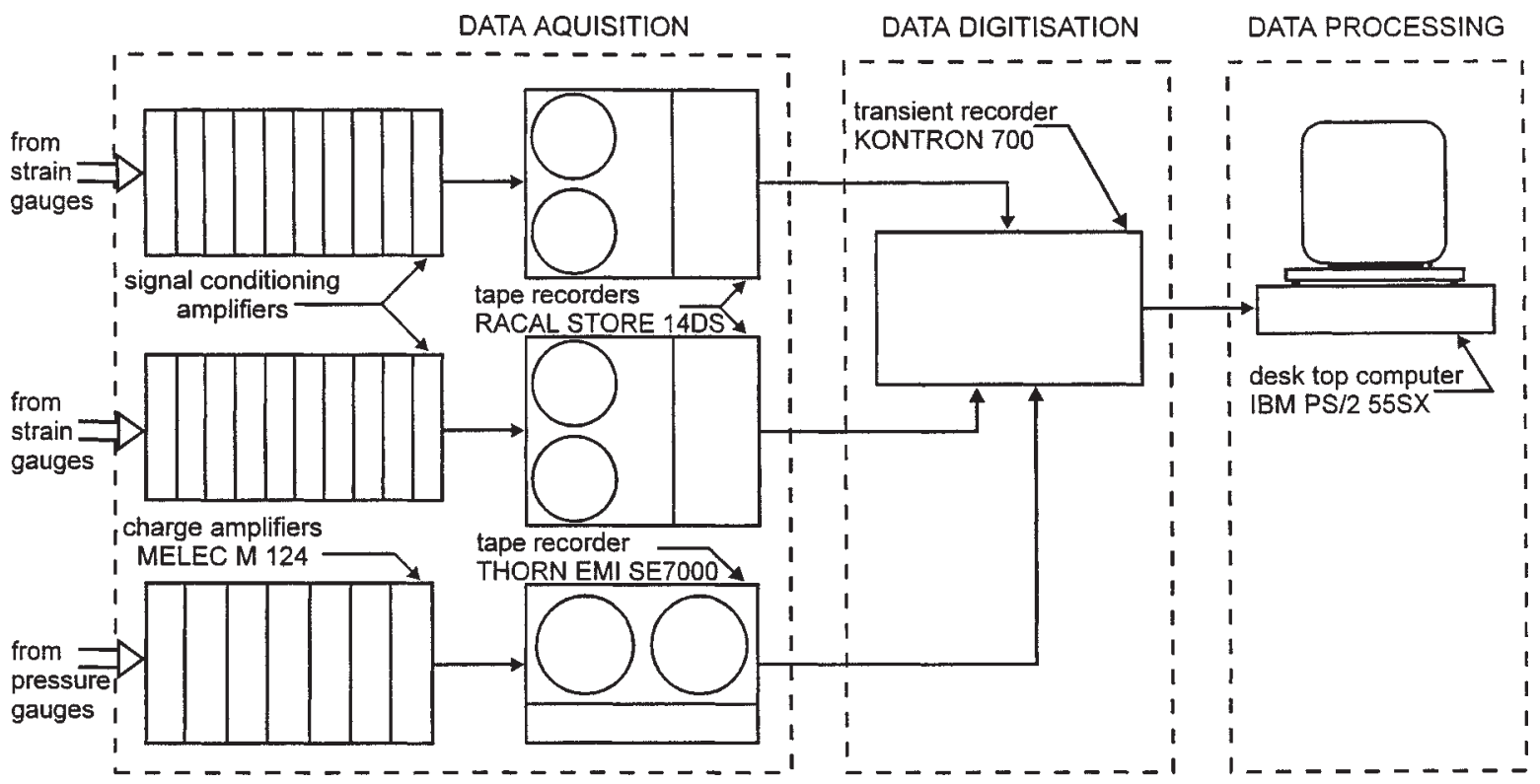

Fig. 5. Data acquisition and analysis instrumentation used in underwater explosion tests.
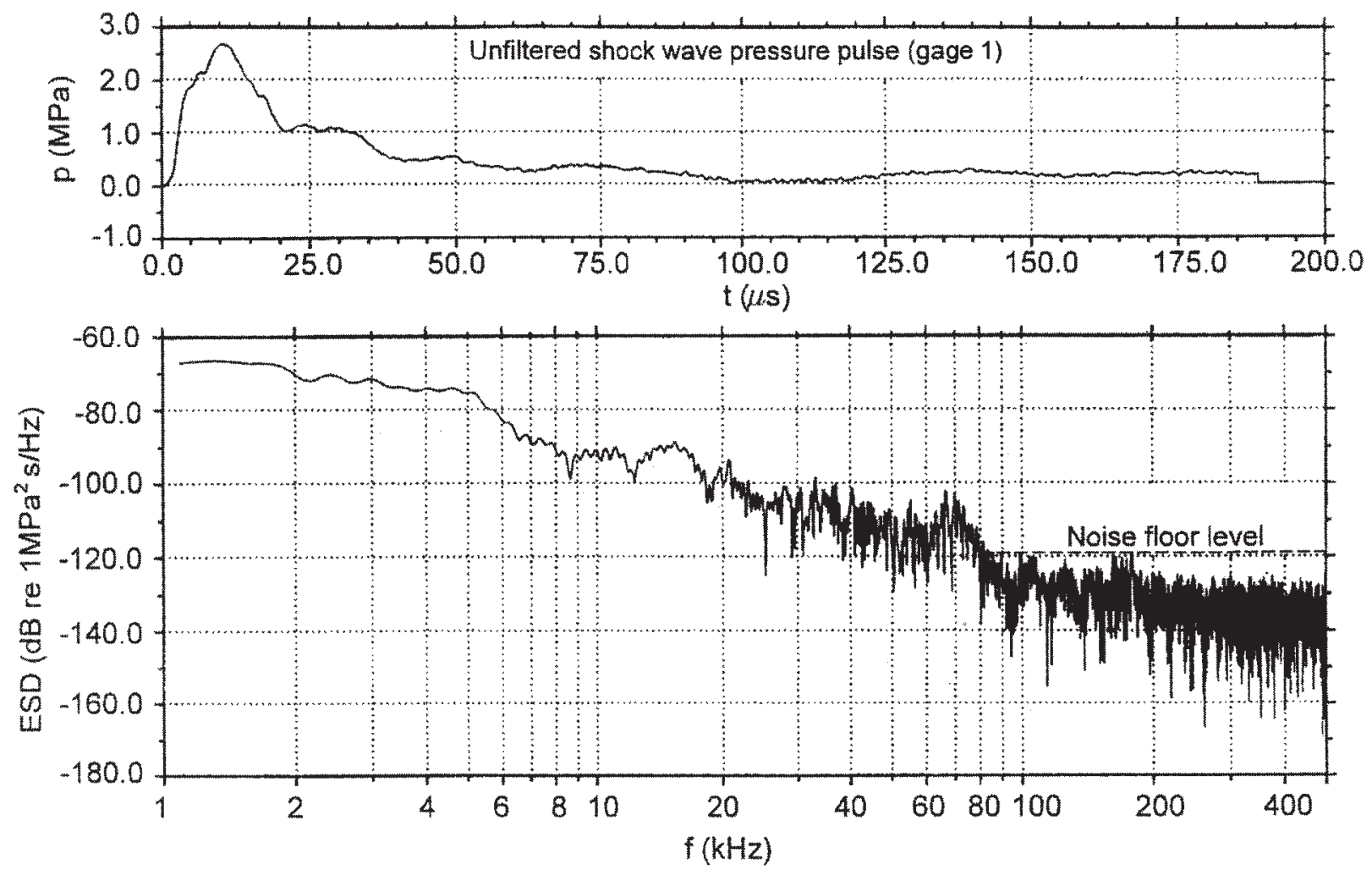

Fig. 6. Energy spectral density of shock wave measured by pressure gage 1 . 

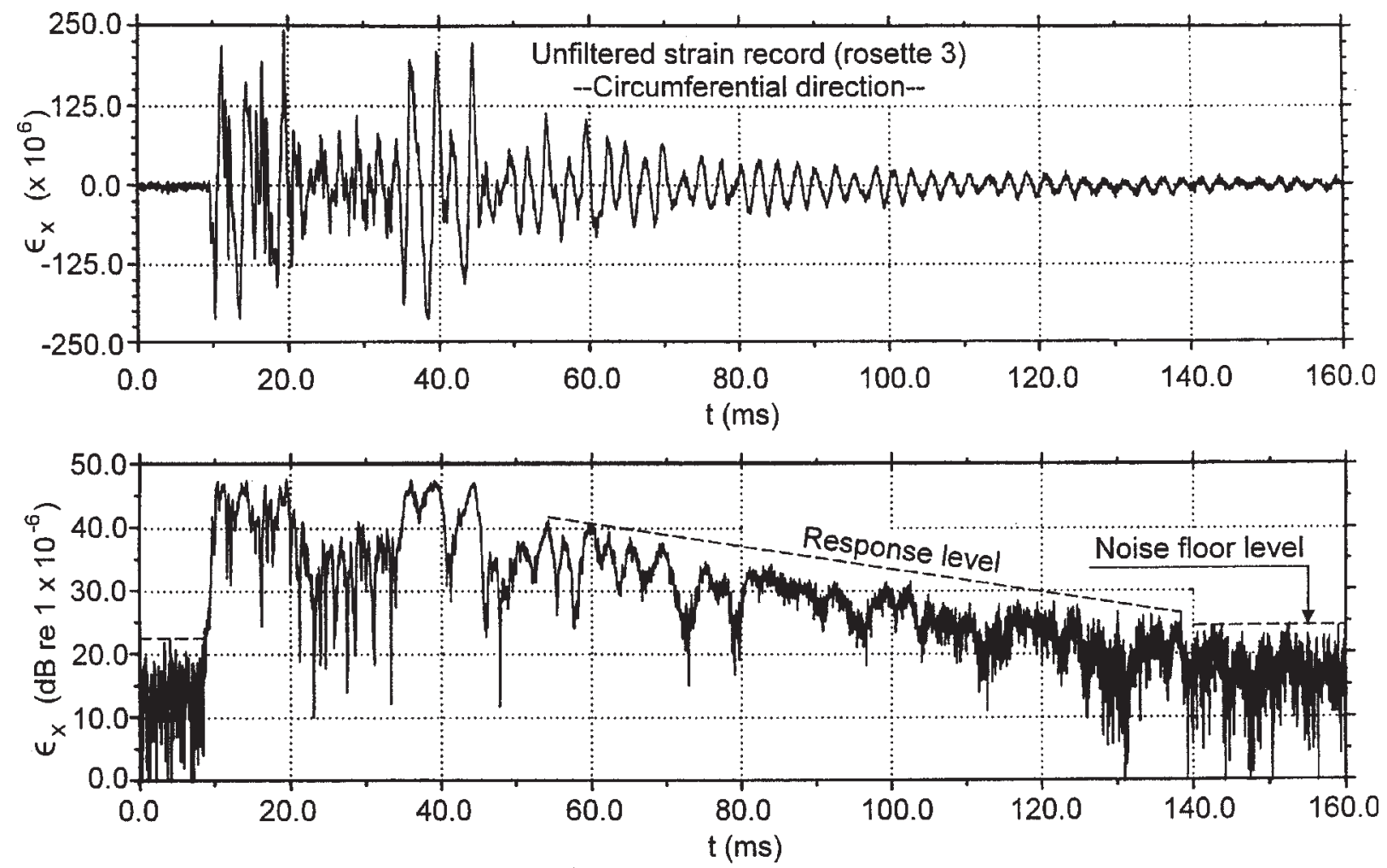

Fig. 7. Magnitude function of a typical strain record (rosette 3) - circumferential direction.

The above operations were applied for each data set of a given transducer in an identical order.

Only the initial part of each pressure signal comprising the shock wave pulse was considered in the filtering process. Discrete pressure-time signals, each formed of 2048 samples, $N$, equally spaced by $100 \mathrm{~ns}$, were transformed to the frequency domain in order to determine the maximum excitation frequency above which any data were considered to be highly contaminated with electrical noise. The ESD (energy spectral density) of the pressure pulse for pressure gage 1, depicted in Fig. 6, indicated that the frequency components above $100 \mathrm{kHz}$ were buried in the noise floor. Consequently, the cut-off frequency, $f_{c}$, to be used in designing a low pass digital filter was equated to $100 \mathrm{kHz}$.

Before analysing the strain signals, with $N=4096$ and $\Delta t=40 \mu \mathrm{s}$, it was necessary to establish that the structural dynamic response had decayed to at least the noise floor level [7], of the pre-triggering level or else exponential weighting would be required. This was achieved by using a Hilbert transform to display the magnitude of a strain signal in the time domain with a logarithmic vertical axis as is shown in Fig. 7. The magnitude function resulting from a Hilbert trans- form represents the positive envelope of the signal under consideration.

It was observed from Fig. 7 that the response level at the end of the time record was below that of the noise floor. Therefore, a procedure similar to that adopted for the pressure signals was applied to the strain records to determine $f_{\mathrm{c}}$ of the digital filter. This was found to be $6 \mathrm{kHz}$.

Figures 8 and 9 show the effect of digital filtering on a sample of a pressure and a strain record, respectively.

\section{Discussion}

\subsection{Pressure records}

Samples of averaged free field filtered pressure records can be seen in Fig. 10. In general, as a pressure gage was positioned further from the explosive charge the maximum value of pressure decreased and the duration of the shock wave increased. The miniature tourmaline pressure gage mounted on the shell surface appeared to capture a higher peak of the pulse impinging the target when compared with the free field gage in 

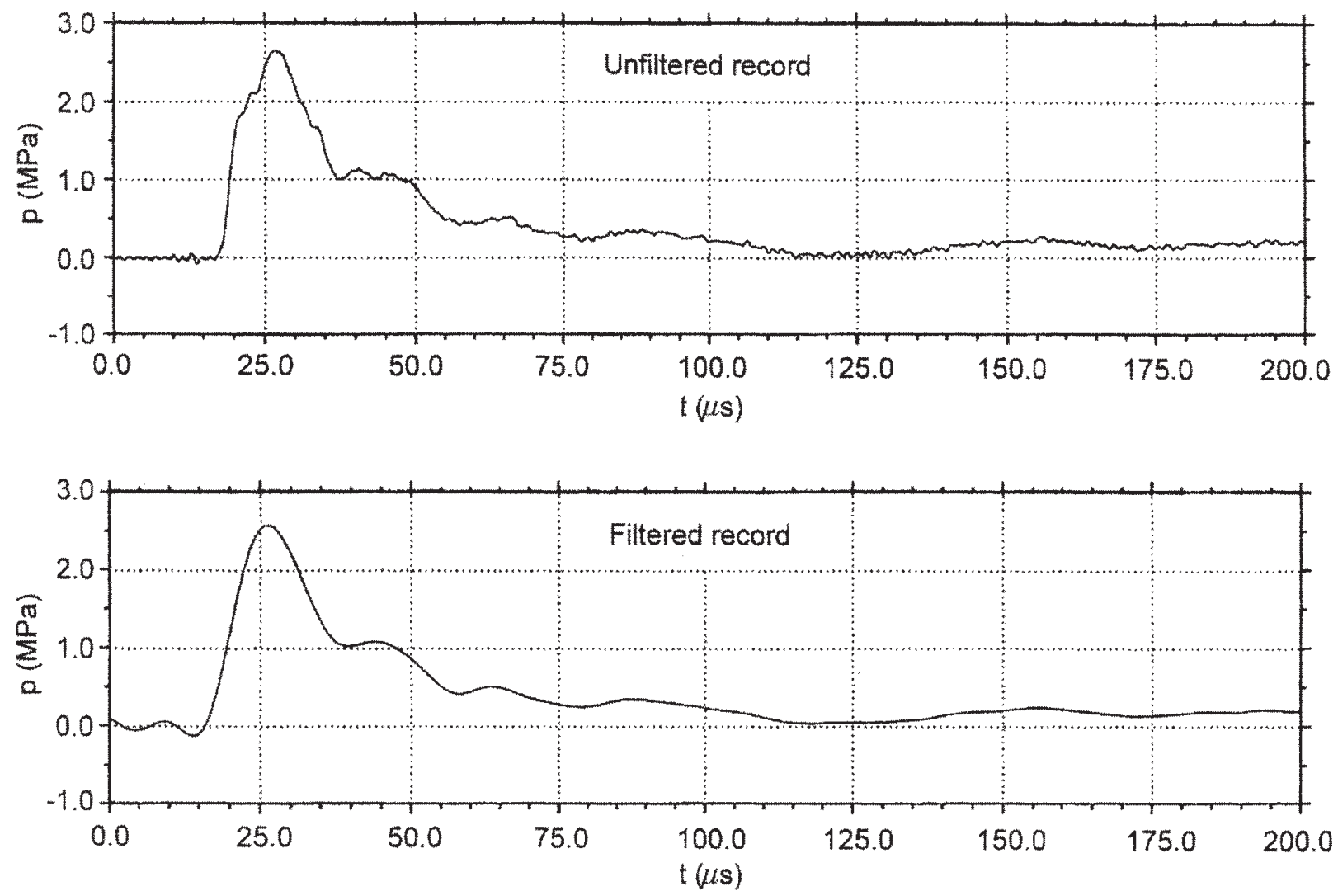

Fig. 8. Effect of filtering on a typical pressure record (gage 1).

the same location $(\approx 16 \%$ higher $)$. This could be explained by the scattering of pressure waves from the target surface which subsequently interfered with the incident pressure wave striking the gage. The induced body motion of the target would influence the build up in total pressure sensed by the tourmaline gage keeping it below what might have been expected in the case of reflections from a rigid surface. Although not shown here the pressure-time record of the tourmaline gage indicated that hull cavitation had taken place.

In many cases of structural damage the effectiveness of a shock wave pulse is dependent in varying degrees on peak pressure, $p_{\mathrm{m}}$, impulse, $I$, and energy flux density, $E_{\mathrm{f}}$, as mentioned earlier.

Cole [2] suggested that the characteristics of shock wave results for a particular explosive can be represented using power laws as follows

$$
\begin{aligned}
& p_{\mathrm{m}}=k\left(\frac{W^{1 / 3}}{R}\right)^{\alpha}, \\
& I=l W^{1 / 3}\left(\frac{W^{1 / 3}}{R}\right)^{\beta},
\end{aligned}
$$

$$
E_{\mathrm{f}}=m W^{1 / 3}\left(\frac{W^{1 / 3}}{R}\right)^{\gamma},
$$

where $W$ is the mass of the charge, $R$ is its stand-off distance, and $k, l, m$, and $\alpha, \beta, \gamma$ are constants.

The above laws can be regarded as fair approximations depending on the range of $\left(W^{1 / 3} / R\right)$. Using the experimentally measured pressure records a linear regression analysis was carried out to determine the constants $k, l, m$ and $\alpha, \beta, \gamma$ for detonator type 79 excluding experimental observations of pressure gages 2, 3 and 8 (see Fig. 11), which were subjected to interference from reflections at the bottom of the tank, the free surface and the test structure, respectively. The resulting characteristic formulae were found to be

$$
\begin{aligned}
& p_{\mathrm{m}}=73.2\left(\frac{W^{1 / 3}}{R}\right)^{1.36}, \\
& I=6124 W^{1 / 3}\left(\frac{W^{1 / 3}}{R}\right)^{0.92}, \\
& E_{\mathrm{f}}=189240 W^{1 / 3}\left(\frac{W^{1 / 3}}{R}\right)^{2.41},
\end{aligned}
$$



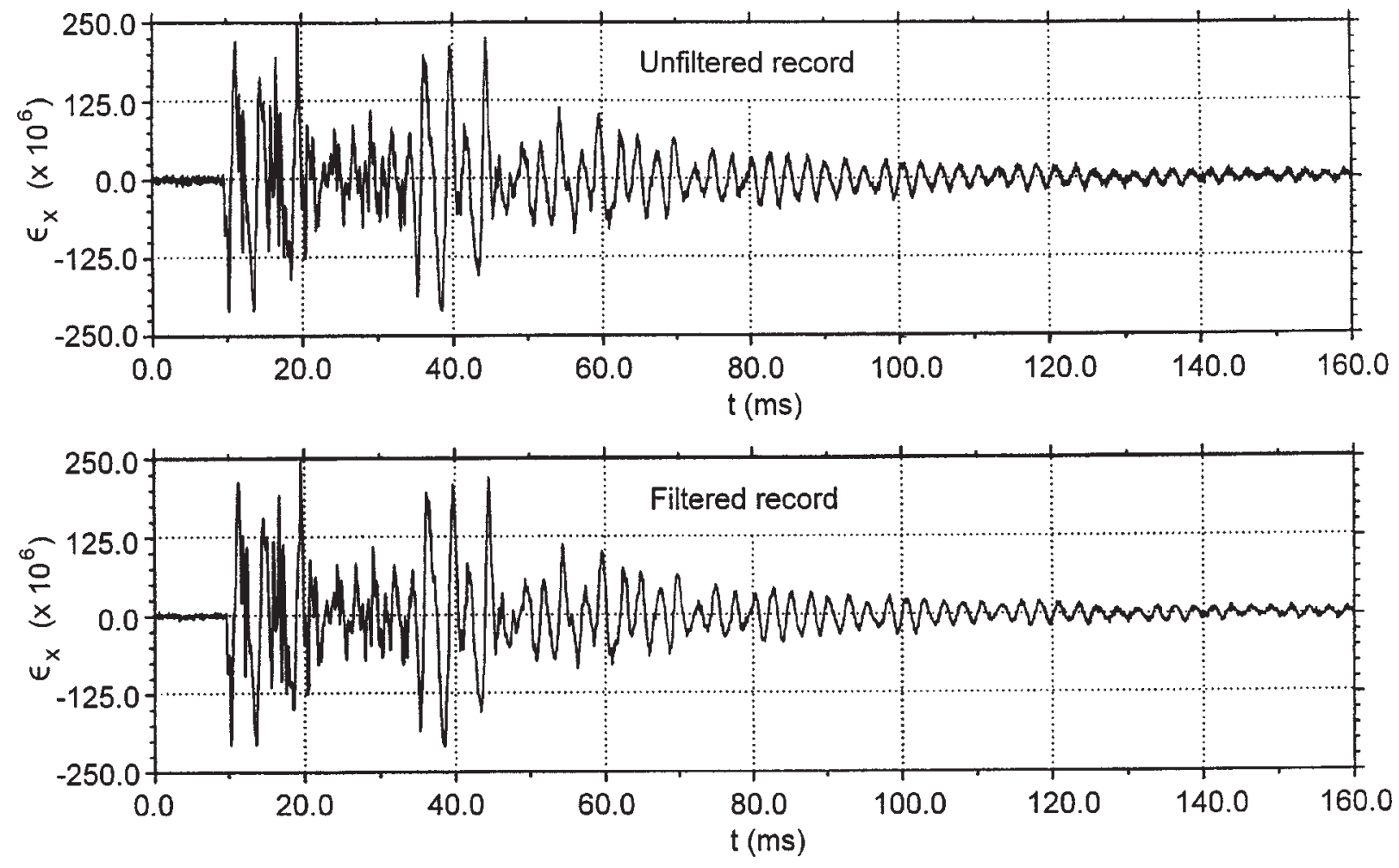

Fig. 9. Effect of filtering on a typical strain record (rosette 3) - circumferential direction.

where $p_{\mathrm{m}}$ is in units of $\mathrm{MPa} ; W$ is in units of $\mathrm{kg} ; R$ is in units of $\mathrm{m} ; I$ is in units of $\mathrm{N} \mathrm{m}^{-2} \mathrm{~s}$; and, $E_{\mathrm{f}}$ is in units of $\mathrm{N} \mathrm{m}^{-1}$.

Shock wave pulse properties $\left(p_{\mathrm{m}}, I\right.$ and $\left.E_{\mathrm{f}}\right)$ were determined utilising the above equations referred to as theory. Table 1 contains a comparison between the predictions and the average experimental observations. The above table indicates that Eqs (4) to (6) are suitable for small charges which were necessary for the tests described above.

In conclusion, empirical formulae were useful in predicting peak pressures of shock waves and this enabled optimisation of the dynamic range setting of the instrumentation used. Additionally, when designing the size of the explosive charge, fired in front of the structure, predictions of the pressure pulse characteristics helped to prevent unplanned structural damage.

\subsection{Strain records}

After the averaging and filtering of strain records the results for each rosette were processed to produce the principal stresses $\left(\sigma_{1}, \sigma_{2}\right)$ a sample of which can be seen in Fig. 12 for $\phi=90^{\circ}$ on meridian M1.
Table 1

Comparison between average experimental observations and predictions of shock wave pulse characteristics along the axis of detonator type 79

\begin{tabular}{|c|c|c|c|c|c|c|}
\hline \multirow[t]{2}{*}{$\begin{array}{c}\text { Pressure } \\
\text { gage }\end{array}$} & \multicolumn{2}{|c|}{$\begin{array}{c}\text { Peak } \\
\text { pressure } \\
(\mathrm{MPa})\end{array}$} & \multicolumn{2}{|c|}{$\begin{array}{l}\text { Impulse } \\
\left(\mathrm{N} \mathrm{m}^{-2} \mathrm{~s}\right)\end{array}$} & \multicolumn{2}{|c|}{$\begin{array}{c}\text { Energy flux } \\
\text { density } \\
\left(\mathrm{N} \mathrm{m}^{-1}\right)\end{array}$} \\
\hline & Test & Theory & Test & Theory & Test & Theory \\
\hline 1 & 2.66 & 2.78 & 70.72 & 67.70 & 67.34 & 61.83 \\
\hline 4 & 1.19 & 1.15 & 34.92 & 37.78 & 14.87 & 13.03 \\
\hline 5 & 0.68 & 0.67 & 25.47 & 26.02 & 5.08 & 5.04 \\
\hline 6 & 0.46 & 0.46 & 20.17 & 20.15 & 3.43 & 2.58 \\
\hline 7 & 0.31 & 0.34 & 17.35 & 16.56 & 1.87 & 1.54 \\
\hline
\end{tabular}

From the above stress records it was noticed that the maximum stresses were experienced by the nearest point on the shell's surface to the explosive charge (i.e., at $\phi=90^{\circ}$ on meridian M1).

The stresses at $\phi=30^{\circ}, \phi=60^{\circ}$ were greater than at $\phi=120^{\circ}, \phi=150^{\circ}$ and this could be attributed to the higher rate of curvature change in the bottom part of the shell's meridional profile, which made the structure appear more stiff in that region. Although the measured strains were greater at the nearest approach to the charge (i.e., at $\phi=90^{\circ}$ ), below this location 

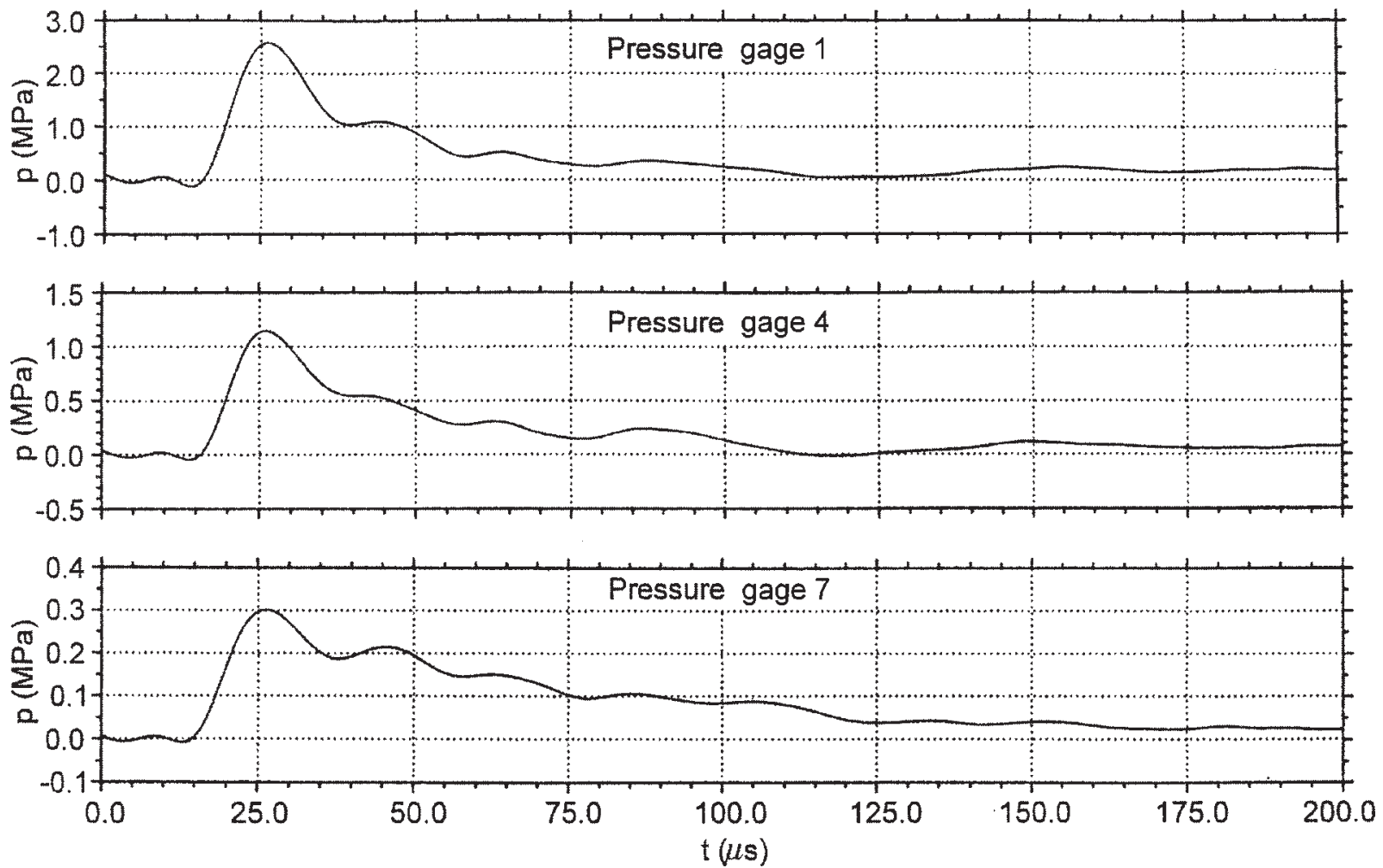

Fig. 10. Average free field filtered shock wave pressure pulse measured at different distances: pressure gage 1, $1100 \mathrm{~mm}$; pressure gage 4 , $2100 \mathrm{~mm}$; pressure gage 7, $5095 \mathrm{~mm}$.

the strains increased, from a lower level, as the base approached. No experimental data were available near the base fixity but extrapolations indicated the greatest strain levels in this region. This supports an earlier conclusion that the lower region of an echinodome shell was a critical zone for design [6].

In all cases, the maximum and minimum stresses were approximately $5 \%$ of the maximum tensile strength of the test structure material (55.4 MPa). It can be concluded that the pressure pulse, generated using $1 \mathrm{~g}$ of explosive charge (detonator type 79) at a standoff of $5.0 \mathrm{~m}$ was too low to invoke the structure's geometrical non-linearity to a degree which could cause serious inaccuracies if it were ignored in the theoretical analysis. In general, in Fig. 12, the principal stresses tended to be on one side of the abscissa because they were the most positive and negative stresses, respectively.

Following the initial shock wave impingement, the first bubble pressure pulse caused a second peak in the experimentally derived principal stresses which was of a similar level to that due to the shock wave.

\subsection{Bubble loading}

As was shown earlier in Fig. 4 for a 1 g explosive charge (detonator type 79) a secondary pulse (first bubble event) occurred $27 \mathrm{~ms}$ after the initial peak. This corresponded to the second peak recorded in the strain-time histories which are not shown here. The time difference between both peaks could be determined from either the pressure records as in Fig. 4 or using the magnitude functions for a strain record as in Fig. 7.

First bubble periods were established to be approximately equal to $27 \mathrm{~ms}$ and $45 \mathrm{~ms}$ for $1 \mathrm{~g}$ and $4 \mathrm{~g}$ explosive charges, respectively. Table 2 contains a comparison between characteristics of both the shock wave and bubble pulses.

From the above table it can be observed that although the peak pressure of the first bubble pulse was much less than that of the shock wave, the area under the pressure-time curve (impulse per unit area) was more than double. This is a clear indication of the importance of considering the effects of the bubble pulse in structural damage. 

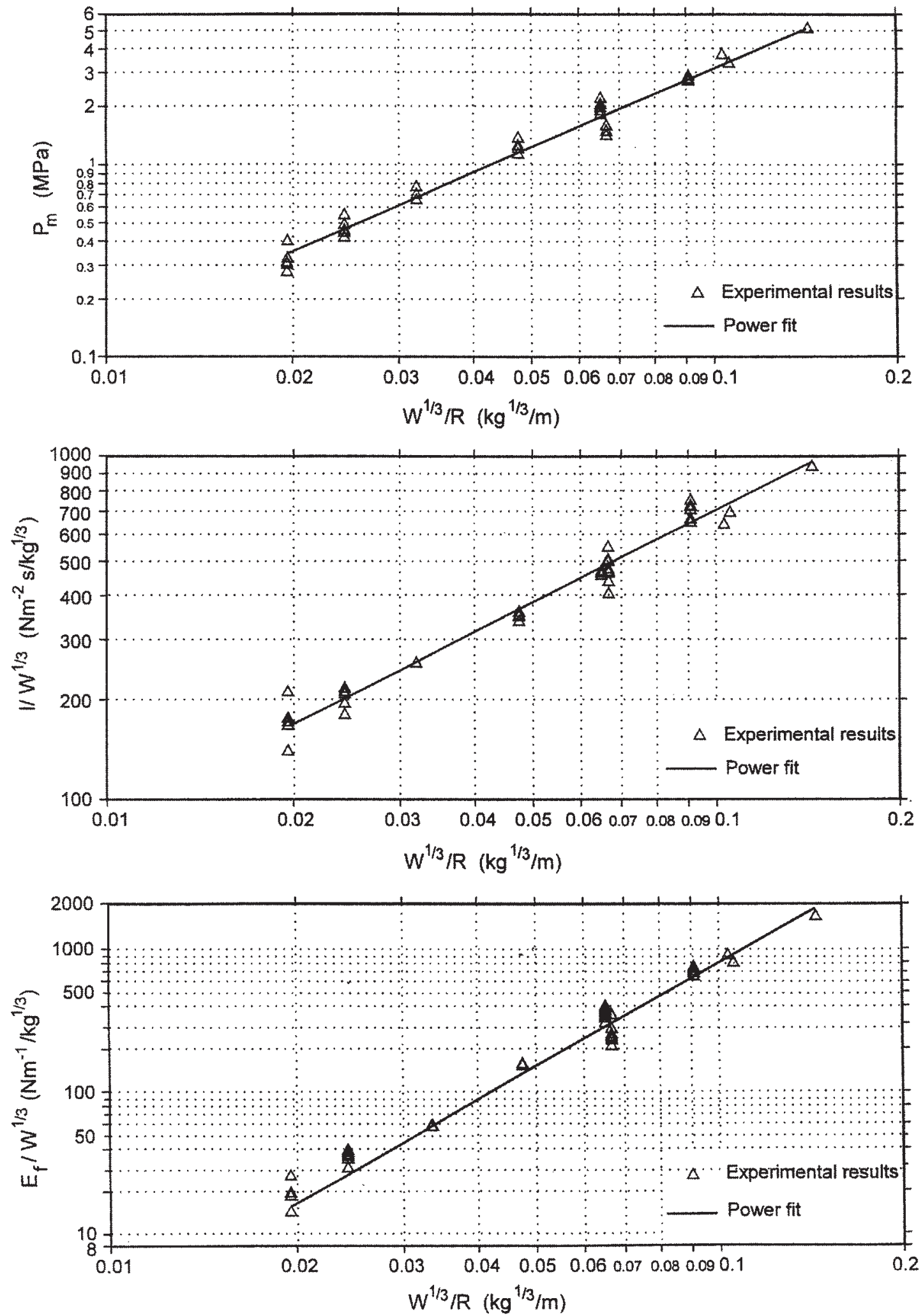

Fig. 11. Fitted models of shock wave pressure pulse characteristics for detonator type 79. 

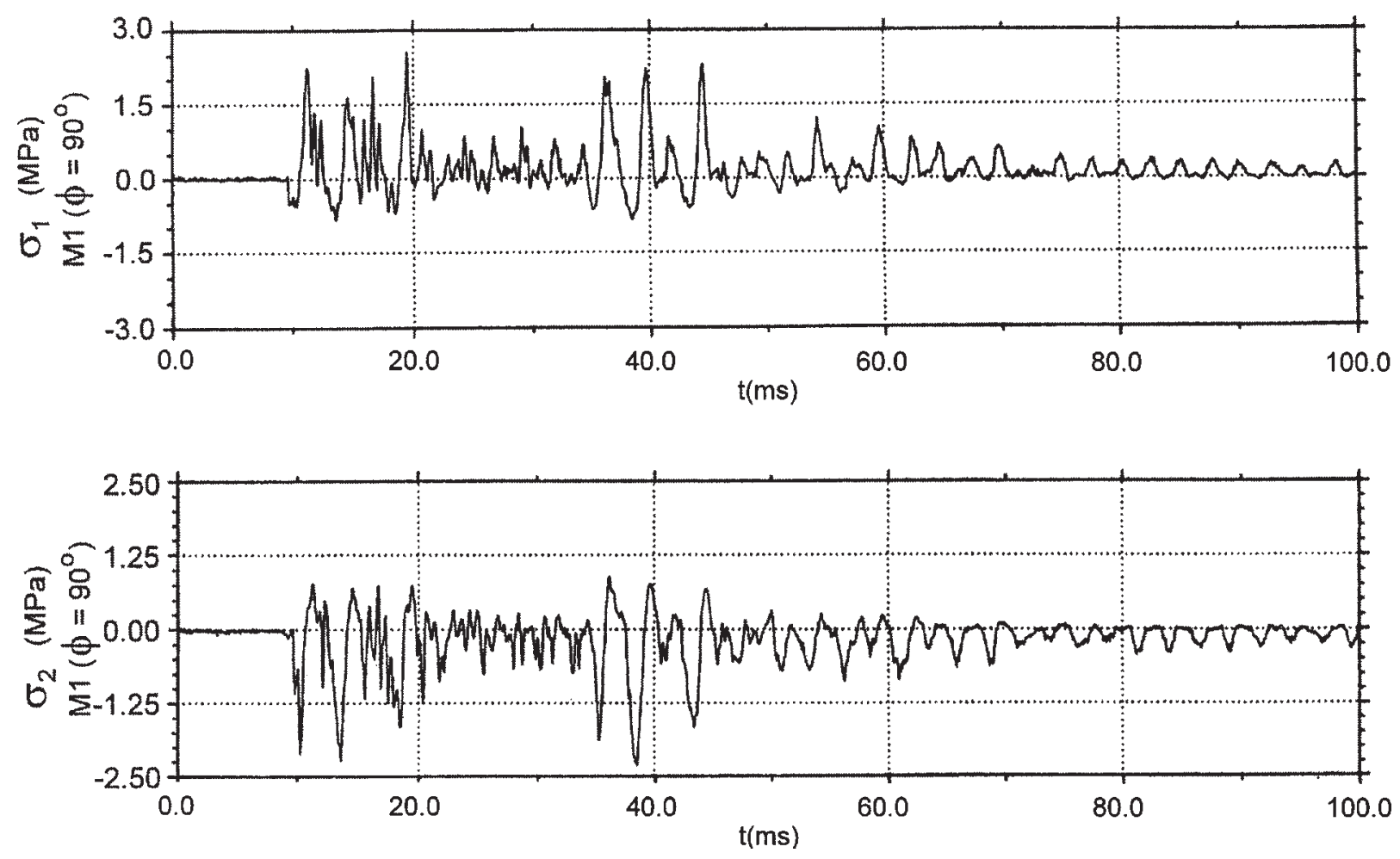

Fig. 12. Experimental principal stresses due to symmetric underwater explosive loading.

Table 2

Comparison between shock wave and bubble pulse characteristics for detonator type 79

\begin{tabular}{|c|c|c|c|c|c|}
\hline \multirow[t]{2}{*}{$\begin{array}{c}\text { Pressure } \\
\text { gage }\end{array}$} & \multirow[t]{2}{*}{$\begin{array}{c}\text { Charge } \\
\text { size } \\
(\mathrm{g})\end{array}$} & \multicolumn{2}{|c|}{$\begin{array}{c}\text { Peak pressure } \\
(\mathrm{MPa})\end{array}$} & $\begin{array}{c}\text { Impulse } \\
\left(\mathrm{N} \mathrm{m}^{-2} \mathrm{~s}\right)\end{array}$ & $\begin{array}{c}\text { Energy flux } \\
\text { density } \\
\left(\mathrm{N} \mathrm{m}^{-1}\right)\end{array}$ \\
\hline & & $\begin{array}{l}\text { Shock } \\
\text { wave } \\
\text { pulse }\end{array}$ & $\begin{array}{c}\text { First } \\
\text { bubble } \\
\text { pulse }\end{array}$ & $\begin{array}{l}\text { Shock First } \\
\text { wave bubble } \\
\text { pulse pulse }\end{array}$ & $\begin{array}{c}\text { Shock First } \\
\text { wave bubble } \\
\text { pulse pulse }\end{array}$ \\
\hline \multirow[t]{2}{*}{1} & 1 & 2.80 & 0.73 & 70.39189 .80 & $68.27 \quad 39.88$ \\
\hline & 4 & 5.08 & 0.99 & 149.15 & 265.36111 .52 \\
\hline \multirow[t]{2}{*}{2} & 1 & 1.78 & 0.54 & 45.43164 .98 & $32.00 \quad 29.80$ \\
\hline & 4 & 3.72 & 0.70 & 100.71242 .49 & $139.68 \quad 79.47$ \\
\hline
\end{tabular}

\subsection{Surface effects}

It was felt that the free field pressure gages performed commendably in measuring peak pressures and hence they were used in determining the effects of free and rigid boundaries on the shock wave. It was established from the pressure records of these gages that at both surfaces, free and rigid, the shock wave was reflected fully with negative and positive magnitudes, respectively.

By knowing the dimensions of the tank together with the pressure gage positions and by determining the time delay of reflected signals the average shock wave speed, $c$, was estimated to be equal to $1418 \mathrm{~m} / \mathrm{s}$. This value would be helpful in any numerical modelling exercise.

\subsection{Simulation aspects}

Drawing upon the experimental data gathered so far, the following numerical approach is proposed in order to facilitate the prediction of echinodome response:

(i) The echinodome structure is to be simulated using conventional thin shell finite elements. The discretisation is to be finer in the zones closest to the charge and in the base region and coarser elsewhere especially in the shadow area.

(ii) Due to the negligible measured strains in the tethering cables these components are not being modelled.

(iii) Measured free field pressure-time history at a $5.0 \mathrm{~m}$ stand-off from the charge is to be prescribed as input loading.

(iv) The semi-infinite fluid is to be represented by the boundary element approach using patch el- 
ements to cover the shell surface and surface of revolution elements to cover the tethering cables.

(v) Reflections from rigid boundaries (side walls and bottom of test tank) and the free surface are to be modelled adopting multiple point sources.

(vi) For early time response prediction the doubly asymptotic approximation (DAA) [4], which takes account of the compressibility of the surrounding medium, is to be employed. By contrast the late time response prediction is to be attempted by means of virtual mass approximation (VMA) [3].

(vii) It is important to validate the numerical model by means of a dry modal test followed by a wet modal test to gain confidence in the simulation of the structural mass and stiffness and the effect of fluid mass.

\section{Conclusions}

1. The maximum strain response was measured on the surface of the structure at its nearest approach to the explosion source.

2. Strain gages located on the lower part of the meridional profile $\left(\phi>90^{\circ}\right)$ indicated an increase in response level as the base of the structure was approached.

3. Realistic structural pressures were measured by the miniature tourmaline gage.

4. Good quality experimental data was obtained suitable for validation of fluid-structure interaction modelling.

\section{Acknowledgements}

The authors are grateful for the assistance and encouragement given by the School of Civil and Environmental Engineering, the University of Edinburgh. The support given by the Ministry of Defence, DERA, Dunfermline is appreciated greatly. Thanks are due to Techni Measure and PCB Inc. for their assistance with the miniature tourmaline pressure gage.

\section{References}

[1] R.H. Cole, Underwater Explosions, Princeton University Press, Princeton, NJ, USA, 1948.

[2] G.A. Corbin and R.C. Reid, Liquid natural gas rapid phase transitions. Report No. GRI 80/0031, Gas Research Institute, Chicago, IL, 60631 USA, 1980.

[3] J.A. DeRuntz and T.L. Geers, Added mass computation by the boundary integral method, Int. J. Numerical Methods in Engineering 12 (1978), 531-550.

[4] T.L. Geers, Doubly asymptotic approximations for transient motions of submerged structures, J. Acoustical Society of America 64(5) (1978), 1500-1508.

[5] R. Royles and J.M. Llambias, Storage aspects of liquid gases underwater and the structural implications, in: Proc. Symposium on Storage and Transport of LPG and LNG, Vol. 2, Koninklijke Vlaamse Ingenieursvereniging Technologisch Institut (Kommissie Metalbouw) Antwerpen, Belgium, 1984, pp. 5572.

[6] R. Royles and K.M.M. El-Deeb, Static buckling appraisal of an echinodome, Experimental Mechanics 33(4) (1993), 263-269.

[7] R.C. Sohaney and Nieters, Proper use of weighting functions for impact testing, Bruel and Kjaer Technical Review 4 (1984), $21-31$. 

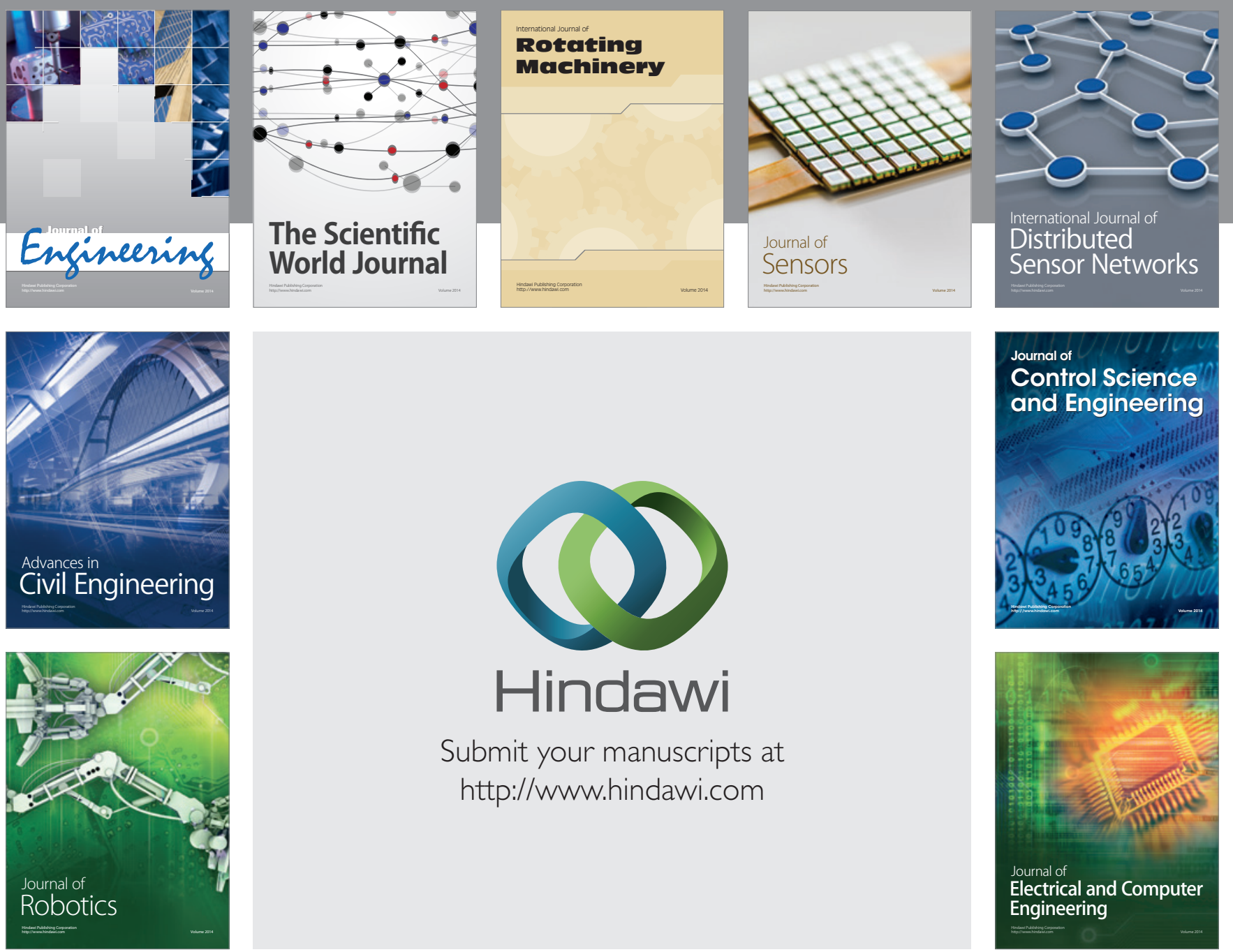

Submit your manuscripts at

http://www.hindawi.com
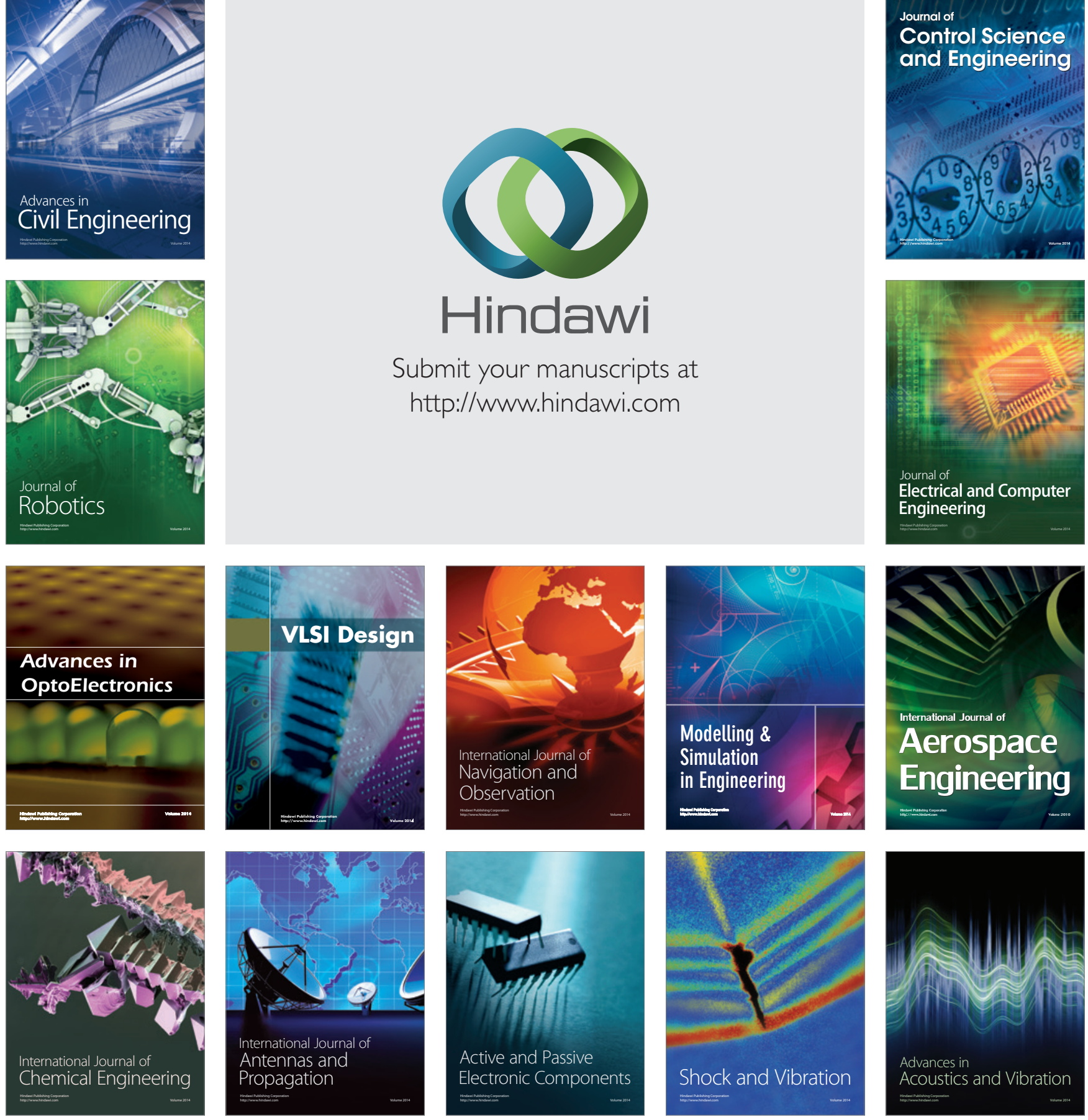